\title{
Virilité et culture ouvrière : pour une lecture des actions spectaculaires de la CGT
}

Isabelle Sommier

\section{(2) OpenEdition}

Journals

Édition électronique

URL : http://journals.openedition.org/conflits/302

DOI : $10.4000 /$ conflits.302

ISSN : $1777-5345$

Éditeur :

CCLS - Centre d'études sur les conflits lilberté et sécurité, L'Harmattan

Édition imprimée

Date de publication : 15 mai 1993

ISSN : 1157-996X

Référence électronique

Isabelle Sommier, «Virilité et culture ouvrière : pour une lecture des actions spectaculaires de la

CGT », Cultures \& Conflits [En ligne], 09-10 | printemps-été 1993, mis en ligne le 13 mars 2006, consulté le 30 mars 2021. URL : http://journals.openedition.org/conflits/302 ; DOI : https://doi.org/10.4000/ conflits.302

Ce document a été généré automatiquement le 30 mars 2021

Creative Commons License 


\title{
Virilité et culture ouvrière : pour une lecture des actions spectaculaires de la CGT
}

\author{
Isabelle Sommier
}

1 Les "actions spectaculaires", périodiquement menées par des militants cégétistes depuis le conflit du Parisien Libéré de 1975, pourraient surprendre dans un pays marqué, à l'instar des autres démocraties occidentales, par l'institutionnalisation du conflit industriel. Symboliques à l'origine, elles se justifieraient par le besoin de "tenir l'actualité", de "faire voir le conflit" lorsqu'il se prolonge sur de longs mois. "Il fallait attaquer sur tous les fronts: sensibiliser l'opinion publique, les pouvoirs publics, et lutter contre le patronat soutenu par le gouvernement. Devant le silence des médias, on a commencé à faire des actions spectaculaires", nous expliqua ainsi l'un des participants au conflit précurseur des ouvriers du Livre ${ }^{1}$. Spectaculaires, les opérations le sont alors sans équivoque : interventions sur le tour de France du 27 juin au 20 juillet 1975, occupation des tours de Notre Dame (6 octobre 1975) ou de la terrasse de la Tour Saint-Denis (17 octobre 1975), blocage de l'autoroute Paris-Bruxelles (30 janvier 1976), occupation du paquebot Le France le 18 mars 1976... Elles dérivent plus tard vers l'action de type commando, avec les affaires de l'usine de roulement à billes SKF à Yvry en juin 1985 ou de l'imprimerie Jean Didier de Massy-Palaiseau durant l'été 1988. Certains ne voient dans ces opérations que le signe de la duplicité "naturelle" des communistes, voire des exercices de "gymnastique révolutionnaire" pour une supposée armée de réserve du parti ${ }^{2}$. Plus sérieusement, on pourrait analyser cette résurgence guerrière comme une conséquence de la routinisation croissante des manifestations syndicales, qui obligerait les organisations à recourir à des actions spectaculaires pour "créer l'événement" et recouvrer une visibilité médiatique qui leur fait toujours davantage défaut. Cependant, cette interprétation n'est que partiellement satisfaisante: si elle met en lumière une condition essentielle de l'émergence des actions spectaculaires de la CGT, elle ne permet de comprendre ni leur forme spécifique, volontiers violente, ni leur dynamique interne, rigoureusement prévue et hautement régulée. Or, ces opérations revêtent toutes des traits réguliers et 
discriminants par rapport aux autres groupes également susceptibles de recourir au spectaculaire. Hormis leur objectif, quel point commun ont-elles en effet avec les initiatives des étudiants en médecine de 1983 ou même avec les actions commandos des paysans? Confrontés aux mêmes mutations de l'environnement, les répertoires d'action évoluent de façon singulière ${ }^{3}$. Ainsi, les actions spectaculaires de la CGT répondent sans doute à une exigence médiatique, mais en empruntant à une histoire et à une culture propres à la classe que le syndicat représente. Par ailleurs, il est douteux qu'elles soient simplement réductibles à un pur souci de visibilité extérieure, dans la mesure où beaucoup d'entre elles échappent aux feux de l'actualité. Ainsi l'occupation d'un train de minerai étranger menée conjointement par les mineurs de Salsigne (Aude) et ceux du gisement d'uranium de Lodève (Hérault) le 22 mai 1990 n'a fait l'objet que d'une photographie commentée d'une simple phrase dans L'Humanitét. Enfin, dernier fait troublant, ces actions peuvent à bien des égards apparaître opposées à la stratégie de séduction de l'opinion publique, en ce qu'elles semblent renouer avec $l^{\prime}$ image des "classes dangereuses" ${ }^{15}$. Parce que ces ruptures de représentations ${ }^{6}$ ne provoquent pas de ruptures du procès d'institutionnalisation, elles posent des questions majeures quant à la signification de la violence et la flexibilité des règles du jeu et de l'ordre public. Nous définirons les actions spectaculaires comme des opérations surprise, menées par un petit groupe dans le cadre d'une mobilisation sociale de plus grande ampleur et qui visent à exercer une pression par une mise en scène ludique et/ou guerrière dirigée contre les symboles de l'adversaire ou, dans une version plus édulcorée, de celui dont est attendue une intervention favorable. De telles actions impliquent donc un degré variable de violence physique avec laquelle elles entretiennent d'ailleurs un rapport ambivalent. En effet, si l'on entend par violence un "comportement visant à causer des blessures aux personnes ou des dommages aux biens"7, les actions spectaculaires ne sauraient être qualifiées de violences de façon générale et systématique. Leurs cibles, strictement matérielles, ne sont volontairement détruites ou endommagées que dans un certain nombre de cas. En revanche, si l'on abandonne le critère de l'intentionnalité de la violence au profit d'une lecture plus dynamique du phénomène, on peut dire que les actions spectaculaires se présentent assurément comme une modalité d'action violente, puisqu'en attaquant le patrimoine patronal (usine, matériel, produits) ou étatique (édifices publics), elles portent matériellement atteinte à autrui et se prêtent à une riposte qui, elle, peut dégénérer en violence ouverte ${ }^{8}$. C'est au cours d'une enquête de type ethnographique menée à l'origine auprès du service d'ordre cégétiste que j'ai été conduite à m'y intéresser. D'une part il s'est avéré que le responsable confédéral et avec lui, des membres de base du service d'ordre, organisaient des actions spectaculaires. D'autre part le personnel d'encadrement des manifestations se recrute précisément dans les catégories socioprofessionnelles qui inclinent le plus vers ce type d'actions: ouvriers du Livre, métallurgistes, mineurs pour l'essentiel, c'est-à-dire les catégories ouvrières traditionnelles. En suivant ce groupe, exclusivement masculin, de militants ou responsables cégétistes, pour la plupart communistes, pendant près de deux ans dans leurs manifestations, en poursuivant le contact deux années ultérieures dans des situations d'interviews formels ou de simples rencontres, j'ai pu récolter de nombreux récits de ces actions, de leur préparation minutieuse, de leur déroulement, des affrontements qu'elles occasionnent parfois. Mais j'ai surtout pu observer l'"esprit de corps" qui les anime et qui véhicule, y compris peut-être de façon mythique, les valeurs constitutives de l'ethos ouvrier : discipline, solidarité dans le combat, glorification de la 
force physique, virilité, etc. Certes quelques chercheurs ont souligné, quoiqu'incidemment, l'importance de cette culture pour comprendre la violence ouvrière, notamment Gérard Noiriel et Patrick Champagne 9 . Mais jusqu'à présent, aucune étude ne l'a réellement intégrée dans une réflexion d'ensemble sur le répertoire $\mathrm{d}$ 'actions. Or, comment en faire abstraction dans l'analyse de la violence ouvrière, sauf à produire une analyse "socio-centrique", qui nie tout l'impact que peut avoir la situation de travail sur l'hexis corporel et, au-delà, sur la "manière d'être au monde"10 ? Cette absence dommageable explique en partie les deux écueils à travers lesquels navigue l'analyse de la violence, considérée soit comme déchaînement irrationnel, soit comme calcul utilitariste. Or, tout l'intérêt d'étudier les actions spectaculaires réside précisément dans le fait qu'elles mobilisent les valeurs constitutives de l'ethos ouvrier au nom de la cause du syndicat ou de la "dignité du travailleur", tout en les soumettant à des contraintes et à des codes leur permettant de rester socialement acceptables. Elles doivent en conséquence être interprétées au regard de la culture ouvrière ${ }^{11}$. C'est pourquoi nous verrons d'abord comment ces valeurs sont intégrées et "retravaillées" dans la préparation et la dynamique des actions spectaculaires pour ensuite avancer une hypothèse explicative de l'ensemble des représentations guerrières données par la CGT. Des valeurs qui guident les initiatives cégétistes, il nous semble que l'essentiel dérive d'une conception d'ensemble de la virilité. Privés de capital économique et de capital culturel, les ouvriers ne disposent en effet d'autres ressources que corporelles. Du prolétaire n'ayant, d'après le sens antique du terme, que sa puissance physique à vendre, il ne sera longtemps renvoyé que l'image d'une pure force musculaire destinée à se soumettre aux nécessités de la technique. Comment ne pas reconnaitre à quel point le travail ouvrier, dans son face-à-face épuisant avec la machine - nous pourrions dire son corps-à-corps, tant le corps se fait appendice de la machine - façonne une identité de groupe fondée sur la force physique ? "Ici, c'est un "homme boeuf" qu'il nous faut", explique un chef des fonderies de Renault, au terme d'une "étude de postes" créée à l'ouverture de l'établissement de Flins $^{12}$. Toute la sociabilité ouvrière est ainsi imprégnée de cette image de soi : la camaraderie masculine sur les lieux de travail ou dans les cercles militants y est rude, franche et virile ${ }^{13}$. Elle s'exprime aisément dans les blagues, souvent douteuses pour l'observateur extérieur, et les coups donnés "en toute amitié" où s'éprouve l'appartenance à la communauté. Pour défendre l'"honneur" ou la "dignité" du groupe - familial, amical ou professionnel - chacun doit être prêt à s'engager dans ces "colères valeureuses"14 toujours susceptibles de basculer dans la "bagarre", sorte de don physique de sa personne à la collectivité. Les conversations de travail tournent volontiers autour des femmes, y compris dans leur version obscène ${ }^{15}$. Les loisirs eux-mêmes, lorsqu'ils ne font pas appel au pur hasard, semblent dirigés vers la dépense de force (sports proprement "physiques", pratiqués plus que dans tout autre groupe social, surcroît de travail manuel par le jardinage ou le bricolage, etc.) ${ }^{16}$. Nous distinguons ici quelques unes des multiples déclinaisons de la virilité qui trouvent à s'investir dans les actions spectaculaires: sens de l'honneur, camaraderie masculine, culte de la force physique et valorisation de la force de combat. Se situant sur le registre de l'exhibition de puissance, les actions spectaculaires, pour différentes qu'elles soient, ont une visée commune : réaffirmer le sentiment communautaire, la cohésion du nous contre les entreprises déstructurantes des autres ${ }^{17}$. A destination des grévistes, ou plus strictement aujourd'hui des activistes, elles répondent d'ailleurs essentiellement à une fermeture d'usine ou à des licenciements de masse ${ }^{18}$. Dans un ouvrage consacré aux luttes très violentes de la sidérurgie lorraine (1979 et 1984), 
Christian de Montlibert a bien montré que "l'intensité de la participation varie avec la transmission et l'inculcation familiale de représentations typiques de la "classe ouvrière" et avec l'investissement dans des associations spécifiques des classes populaires"19. Dans et par les actions spectaculaires, c'est la fibre identitaire, la "conscience fière" du mouvement ouvrier qui est ainsi stimulée par la réactivation des valeurs constitutives de l'ethos ouvrier. Elles relèvent d'abord, dans leur objectif même, de la défense de l'honneur et de la dignité du groupe, menacé dans son emploi ou son identité par des politiques de rationalisation du travail, de restructurations industrielles ou autres licenciements. Cette perspective est dans tous les esprits des protagonistes : ouvriers du Livre engagé dans le conflit du Parisien Libéré qui refusent $\mathrm{d}^{\prime}$ "abdiquer [leur] dignité de travailleur"20, dockers en lutte pour le maintien de leur statut, sidérurgistes lorrains ${ }^{21} \ldots$ Face aux dizaines de licenciements annoncés par la direction de Renault-Cléon en 1987, Robert H. ne voyait qu'une issue, "réagir, ne pas se laisser faire", faute de quoi l'initiative patronale n'aurait été qu'une "première charrette de licenciements massifs" ${ }^{22}$. On peut légitimement se demander si certaines opérations ne relèvent pas purement et simplement de cette volonté de marquer le coup, de résister jusqu'au bout, de relever la tête une dernière fois malgré la certitude d'une cause perdue d'avance. Un seuil semble avoir été franchi, qui autorise les grévistes à ne plus jouer le jeu bien réglé des relations industrielles et à riposter à ce qu'ils considèrent être une offensive, une ouverture des hostilités par le patronat, dans une même logique de guerre. Les actions spectaculaires ou "actions coups de poing" s'inscrivent aussi et, peut-être surtout, dans la culture de camaraderie masculine. "On est bons potes ensemble, même si on s'accroche parfois, même politiquement, mais on reste néanmoins ensemble et quand il y a quelque chose, on sait faire le carré et on y

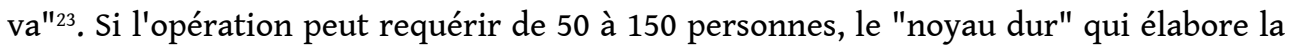
tactique ou qui participe à toutes les initiatives est, lui, beaucoup plus restreint. L'invention permanente de nouvelles actions appelle d'abord l'ingéniosité et la fantaisie du groupe : "On avait un slogan au Parisien Libéré : "La lutte est dure mais pas triste" (...) Chercher des actions qui peuvent faire parler de nous, un truc inhabituel où on berne et les flics et les pouvoirs publics. C'étaient des trucs surprise; il y avait un côté un peu drôle de faire cela"24. La dimension ludique est en effet essentielle à ces opérations, qu'elle se traduise par le côté un peu folklorique de leur préparation ou par leur dynamique de farce qui s'accorde alors avec la "culture tonique" et rude de la classe ouvrière, mise en lumière par Michel Verret ${ }^{25}$. Les symboles de la domination sont défiés, attaqués et soumis dans la liesse. L'esprit frondeur se traduit dans le privilège des opérations de défi, où la toute-puissance de l'adversaire se trouve soudain prise en dérision: "berner les flics", "prendre" un territoire interdit, déménager symboliquement le siège du pouvoir patronal, etc. Jean-Pierre B. raconte à sa manière l'occupation de la préfecture de Rouen en 1987 : "On les a complètement blousés. On avait organisé ça d'une façon, je dirais, un peu militaire. On avait un groupe de camarades qui étaient entrés à la préfecture avec des pancartes qui étaient planquées sous eux et des chaînes. Et il y avait un autre groupe qui, lui, était chargé de faire diversion devant la préfecture en brûlant des pneus. Ensuite, il y avait un troisième groupe qui arrivait en car pour manifester devant. Tout ça s'est parfaitement enchaîné (...) Là, on les a complètement blousés. Toute la matinée. Ils savaient plus où donner de la tête. L'officier des RG qui était chargé de nous suivre, parce que forcément ils sont toujours dans nos pattes dans ces conflits-là, on l'avait blousé..." ${ }^{26}$. Lorsqu'elles visent l'entreprise (locaux, matériel ou produits), les actions spectaculaires s'apparentent 
parfois aux phénomènes d'inversion de l'ordre et de l'autorité observés dans les carnavals ou dans certains rites. Les ouvriers se réapproprient leur monde, leurs biens et les transforment à leur guise. Ainsi l'emprunt, en août 1985 de 50 automobiles Renault à Gennevilliers, pour réaliser une "opération escargot" sur les Champs Elysées, territoire traditionnellement interdit aux manifestations ouvrières. Le baroud d'honneur était double : contre la direction et contre les pouvoirs publics. La dimension ludique apparaît au résultat dans des réflexions de type "on les a bien eus", "Qu'est-ce qu'on leur a mis !", "On a bien rigolé" et plus tard, dans les récits épiques qui ne se priveront pas d'accentuer le ridicule ou le désarroi des vaincus. Les actions spectaculaires requièrent un groupe hautement discipliné et encadré, une préparation minutieuse qui n'est pas sans rappeler les "ficelles" classiques des romans populaires d'espionnage: l'un sera chargé de photographier le lieu choisi, l'autre agrandira le tirage pour permettre au "chef " d'élaborer une tactique et de distribuer les rôles, tel autre enregistrera les horaires et les habitudes du personnel de surveillance, etc. "Pour faire le train [de minerai étranger] il a fallu cinq jours pour le suivre et savoir où il passe, les heures de passage en gare, etc." ${ }^{27}$. La fonction identitaire est encore plus éclatante ici, puisque le succès de l'opération dépend de la cohésion d'un petit groupe fortement structuré et hiérarchisé, de sa capacité à "tenir le secret" et à obéir. L'action en elle-même ne manque pas de rocambolesque et met en scène, de façon somme toute peu coûteuse, une stratégie d'attaque révolutionnaire contre l'adversaire de classe. Ainsi, loin de se déployer sans retenue sur le terrain physique immédiat, les actions spectaculaires privilégient la mise en scène et deux types de dispositions ouvrières : la disposition au jeu et la disposition à la remise de soi $^{28}$. Mais elles n'oublient pas les prouesses physiques qui, même si elles ne sont pas effectives, sont valorisées de façon presque mythique par le type d'opérations et la perspective, toujours possible, d'affrontements avec la police. Ainsi ce responsable cégétiste qui nie l'existence d'"armes", au motif que "c'est des gars costauds" dont certains "ont fait des exploits dans la Résistance" et qui par conséquent "n'en ont pas besoin"29. Le groupe ouvrier se présente de la sorte solidaire, courageux, résistant, héroïque et fort. Le culte de la force physique dans la classe ouvrière relève du processus classique de retournement du stigmate. C'est en effet au moment même de l'expansion industrielle que se transforme l'iconographie socialiste: le corps féminin, figure allégorique du mouvement révolutionnaire de 1830 à 1848 , est progressivement délaissé au profit du torse nu du travailleur manuel ${ }^{30}$. Cette représentation, qui se perpétuera ensuite dans la culture communiste issue de la Révolution de 1917, n'avait rien de réaliste. Mais elle met l'accent sur le travail physique comme critère d'appartenance de classe et, au-delà, participe à une tradition populaire d'héroïsation du corps ${ }^{31}$. Il est frappant de constater à quel point l'antagonisme de classe peut s'ancrer dans l'image du corps de l'Autre. La représentation de la classe ouvrière comme "classe dangereuse" n'est pas sans rapport en effet avec celle d'une masse corporelle lourde, brutale, presque animale car ne trouvant à s'employer, hors travail, que dans la débauche. Georges Vigarello a montré à ce propos combien, dans la seconde moitié du siècle, les débats des hygiénistes sur le "maintien des corps" ouvriers reposaient sur une double exigence: utilitariste (adaptation des corps au travail dans une perspective d'accroissement de la rentabilité) et moraliste (domestication des corps pour lutter contre les dépravations) ${ }^{32}$. Laid voire repoussant et féroce, tel apparait le physique ouvrier aux yeux des bourgeois du XIXème siècle qui, de leur côté, sont montrés ventrus et pervers dans les caricatures populaires ${ }^{33}$. "Ainsi les luttes politiques et sociales se traduisent-elles notamment dans 
un conflit des apparences où le visage de l'autre livrerait dans l'amplification grotesque du détail visible la nature cachée de sa moralité corrompue" ${ }^{34}$. Bien qu'euphémisées, ces représentations demeurent sans doute encore prégnantes aujourd'hui. Forts, insensibles à l'effort et à la douleur, peu intelligents, sont autant d'attributs qui restent encore aujourd'hui souvent attachés aux ouvriers ${ }^{35}$. Mais ceux-ci partagent également des représentations collectives dépréciatives de leurs adversaires, qu'un simple exemple nous permettra d'éclairer. Lors du conflit de Renault-Cléon d'octobre 1991, les responsables syndicaux cégétistes distribuèrent des douzaines d'oeufs aux grévistes qui les lancèrent contre les cadres de l'entreprise. En substituant ainsi le mou au dur, l'oeuf aux boulons ou aux pierres, ils exercèrent de façon indéniable une fonction pacificatrice. A les écouter pourtant, l'un d'entre eux nous fit une réflexion d'un grand intérêt pour le sujet qui nous préoccupe : "A la limite, c'est plus humiliant pour celui [N.B. un cadre] qui prend un oeuf [plutôt qu'un boulon], comme par exemple pour un homme, prendre une gifle, c'est plus humiliant que prendre une châtaigne" ${ }^{136}$. Notre interlocuteur semble ainsi doublement ravaler le cadre à un rang de masculinité inférieur à l'ouvrier: d'une part, en opposant implicitement, avec la conjonction "comme", le cadre et l'homme ; d'autre part, en comparant les humiliations respectives majeures pour un cadre - des oeufs, matière flasque et dégoulinante qui tâche leurs complets - et pour un homme de l'autre - coup direct donné en face à face et sans médiation. L'action se charge d'exprimer de la sorte un mépris profond de l'ouvrier contre le "moindre homme" - voire le "faux" homme - que serait le cadre. Certes, ces propos ont tout de la rationalisation militante a posteriori mais ils témoignent aussi de l'alliance inconsciente établie entre le travail manuel et la force physique, la force physique et la virilité, la virilité et le courage. La disposition à un comportement violent engage en effet un certain rapport au corps et à sa puissance ${ }^{37}$. L'affirmation identitaire des dominés par la violence joue d'autant plus dans nos sociétés où l'hexis corporel légitime passe par toujours davantage de retenue, de maîtrise de soi, d'autocontrôle des émotions et par la résolution verbale et policée des conflits. Où la force physique elle-même est moins requise dans le procès de travail. Où l'on assiste au "passage d'un gestuel universel de travail à un gestuel universel de contrôle" ${ }^{38}$. Dans ce cadre, la posture virile contribue à la construction d'une image de soi valorisante et distinctive en ce qu'elle s'oppose à la fois à la féminité et à l'ordre dominant. A ce propos, il est intéressant de noter que déjà, Georges Sorel exhortait la classe ouvrière à l'héroïsme, au "sublime", par la violence, sans doute dans une perspective stratégique, mais en des termes sans équivoque du dégoût qu'il portait à la "dégénérescence" de la bourgeoisie, dégénérescence provoquée par sa "niaise" aspiration à la paix et à la conciliation. La violence prolétarienne se devait à ses yeux de "marquer la séparation de classe" en même temps que d'enrayer la décadence de la société39. Mais le culte de l'homme fort, viril a également une efficacité politique en ce qu'il symbolise la fierté et la combativité du groupe. Le retournement du stigmate est ainsi complet puisque la force physique requise dans un procès du travail aliénant et en partie responsable de représentations sociales négatives se mûe en force de combat au service de la justice. Ainsi du mineur qui, au lendemain de la Résistance, devient la figure emblématique du Parti Communiste Français : "Courageux, héroïque, solidaire, discipliné, responsable, viril et indomptable, le mineur, loin d'être écrasé par le destin, le transforme en avenir radieux" $^{40}$. Comme le souligne Pierre Bourdieu, "une classe qui, comme la classe ouvrière, n'est riche que de sa force de travail ne peut rien opposer aux autres classes, en dehors du retrait de cette force, que sa force de combat qui dépend de la force et du 
courage physiques de ses membres et aussi de leur nombre, c'est-à-dire de leur conscience et de leur solidarité ou, si l'on préfère, de la conscience de leur solidarité" ${ }^{41}$. Force de travail et force de combat sont intimement liées dans la culture ouvrière. Pour Michel Verret, la familiarité de la classe ouvrière à un quotidien fait de "situations limites" (de chocs, d'effort, de fatigue) expliquerait la "forme vertige" que revêt une culture où s'exprime une certaine séduction de la mort ${ }^{42}$ : alcool, sports de combat, etc. Cette idée $\mathrm{d}$ '"aller à la limite" amène de son côté Olivier Schwartz à emprunter à Georges Bataille la notion de "conduites de dépense", comme "mode d'usage des forces qui repose sur l'effort pour consommer celles-ci au maximum" ${ }^{43}$. Travailler, frapper et boire relèveraient ainsi "d'un même modèle: celui du déploiement non inhibé des forces" ${ }^{44}$. Le travail ouvrier active et réactive ainsi "une figure traditionnelle du masculin (...) qui associe électivement à la virilité le risque, le conflit et le défi" ${ }^{45}$, selon le principe du "cogito viril : je m'expose, donc je suis"46. Justicières, de leur point de vue, les actions spectaculaires tiennent à la fois de deux modèles qui engagent, à des degrés variables, la cohésion du groupe et son courage physique : Robin des Bois par un certain côté bon enfant, comme l'opération "Murons la répression" conduite à RenaultBillancourt le 27 août 1985. Un petit groupe entreprit alors de mettre "hors d'état de nuire les bureaux de la répression" (entendons ici les bureaux du service des relations du travail), en condamnant la porte d'entrée avec parpaings et mortier, avec le chef de service et ses collaborateurs enfermés à l'intérieur. Mais elles rappellent aussi certaines formes de guérilla urbaine lorsqu'elles lancent un assaut contre une entreprise, a fortiori lorsque celle-ci est occupée par les forces de l'ordre, comme ce fut le cas de l'entreprise SKF le 5 juin 1985. Ce jour-là, un groupe d'une quarantaine de personnes, 150 selon la CGT, investit l'usine à l'aube, en défonçant un mur à l'aide d'une benne. Armé de barres de bois, de billes d'acier et de frondes, il finit par s'installer sur les toits avec drapeaux rouges et tricolores. Pour une fois, des femmes étaient présentes, mais elles jouèrent le rôle, conforme à la division traditionnelle des rôles, d'infirmières pour les "guerriers" blessés : "A SKF, il y avait des copines pour soigner les yeux contre les lacrymos; ça, c'est très important" ${ }^{47}$, nous fut-il dit en signe de "modernisation" du groupe. L'esprit de résistance de la communauté se trouve ainsi magnifié par l'esprit de sacrifice de quelques uns qui n'hésitent pas à "payer de leur personne" au nom de la dignité du nous et qui rejoindront peut-être le martyrologe populaire. En témoigne ce récit des ouvriers du Parisien Libéré : "Malgré les visages de plus en plus tirés, émaciés par la fatigue, les yeux rouges de sommeil perdu, malgré les dépressions, les coeurs qui se fatiguent, surtout la nuit où il faut courir, grimper, se cacher, craindre, espérer, se voir humilié et mis au rang de délinquant; malgré tout cela, devant l'inconcevable attitude patronale et gouvernementale, chacun recommencera la nuit suivante pour faire entendre son droit de vivre comme tout le monde, son droit au travail. Mais aussi avec l'espoir de retrouver une vie normale et des enfants qui ne soient plus perturbés par un père "gréviste depuis 17 mois", qui donne chaque nuit un peu de sa santé, risquant même sa vie et plus tard... qui sait... peut-être la prison pour n'avoir jamais voulu abdiquer sa dignité de travailleur"48. La dramatisation des propos pourrait donner à ne voir dans ces actions coups de poing qu'un simple effet d'hystérésis, un décalage entre des représentations collectives idéalisantes et la réalité objective du statut ouvrier. Dans cette perspective, elles reproduiraient et mettraient en scène le mythe de l'ouvrier en lutte contre l'injustice, perpétuellement pourchassé par la coalition des puissants. Mythe qui d'ailleurs peut être inconsciemment renforcé à des fins identitaires : dans une société où sont chaque jour un peu plus dépréciées, non 
seulement les valeurs de masculinité, mais aussi la force physique dans le travail industriel, où la force de combat peut difficilement s'investir dans un projet politique, l'affirmation identitaire se réduirait, pour ces militants ouvriers, à une théatralisation de la force de combat. On rejoindrait la "nostalgie de l'épreuve" décrite par Finkielkraut, nostalgie de "l'épreuve virile comme critère d'identité" ${ }^{49}$. Compte-tenu pourtant de l'attention avec laquelle les responsables syndicaux s'emploient à retravailler les dispositions du groupe, valorisant les unes - esprit de dérision, soumission à la discipline collective, solidarité-, soumettant les autres à une symbolisation croissante - culte de la force physique, goût pour la bagarre -, une autre hypothèse est concevable, qui intégrerait dans un même schéma explicatif l'ensemble des représentations guerrières donné par la CGT. A l'instar du service d'ordre des manifestations, les actions spectaculaires ou "actions coups de poing" offriraient aux dispositions guerrières un espace de satisfaction sous contrôle. Elles permettraient ainsi de prendre en charge certains ouvriers ou des grévistes désespérés, en les encadrant et en les soumettant à l'action pédagogique et canalisatrice des responsables syndicaux. "Il faut que la colère s'exprime, explique le secrétaire adjoint pour la sidérurgie R. Huin (CFDT) aux lendemains de la première attaque du commissariat de Longwy le 30 janvier 1979, mais pas de n'importe quelle façon. Il faut éviter la violence physique, car elle pourrait se retourner contre les intérêts qu'il s'agit de défendre" ${ }^{50}$. La médiation syndicale et la préparation des actions spectaculaires semblent se voir ainsi assigner la fonction de transformer une violence colérique, pulsionnelle et spontanée, et à ce titre propice aux incidents, en violence instrumentale, calculée, froide et orientée vers une fin définie ${ }^{51}$. "On explique aux copains, quand on arrive à cette phase de lutte : voilà les conneries qu'il ne faut pas faire. Des conneries, on en a faites. On a eu un aveugle parce qu'il y a eu une connerie de faite. Parce que, même chez nous, il y a des gens qui veulent toujours en faire plus, qui se considèrent intouchables. "C'est pas vrai, la police elle peut pas tirer. Le gars qui est au chômage et qui accepte de faire le boulot d'un gréviste, "il faut le tuer". Des choses de ce genre, ça a existé. Et on avait des copains qui veillaient justement à ce qu'il n'y ait pas de débordements de cette nature" ${ }^{152}$. Soumises, durant les manifestations, à l'action pédagogique et canalisatrice des responsables syndicaux, les valeurs constitutives de l'ethos ouvrier sont mobilisées au service des intérêts supérieurs de l'organisation : déroulement pacifique, respect de l'ordonnancement prévu... Par les actions spectaculaires, elles trouvent en outre une enceinte d'expression hautement policée et régulée où la force physique se déploie, parfois à grands renforts dramaturgiques, précisément pour ne pas se concrétiser. Leur dimension guerrière ne saurait en effet être surestimée : après l'échec de "la stratégie SKF", la CGT n'ira pas au-delà dans l'escalade guerrière et reviendra vers des actions plus symboliques dirigées contre les biens de l'entreprise. C'est notamment l'objectif d'une nouvelle forme d'action directe, la "mise sous surveillance", qui consiste à bloquer la poursuite d'une restructuration industrielle en déménageant les machines ou le matériel. Elle fut utilisée à Renault, Alcatel-Thompson (Colombes), Wonder à Saint-Ouen, etc. Du point de vue cégétiste, leur préparation minutieuse et leur encadrement préviendraient les risques de dérapages ou d'incidents qui, en l'absence de responsables syndicaux, ne manqueraient pas de survenir : "Pendant le conflit du Parisien Libéré, on a fait des choses extrêmement violentes mais on les a faites d'une manière telle qu'on n'a jamais eu de problèmes. Le pépin qu'on a eu, c'est des gars qui voulaient jouer les zorros tout seuls, et ils sont passés carrément au-dessus de ce qu'on avait dit." ${ }^{53}$. Pour le train de minerai nigérien occupé le 22 mai 1990 par des mineurs, 
"il avait été décidé de ne pas répandre les bidons en dehors du train parce qu'il y a de l'uranium dedans. On a commencé à cinq heures du matin, vers seize heures l'aprèsmidi, on a eu beaucoup de mal à dire aux gars "ne le faites pas". Sûrement qu'on en aurait parlé cent fois plus, mais en rendant les travailleurs responsables d'une catastrophe écologique. C'est difficile d'expliquer aux gars. Le gars, il dit "moi, j'ai plus de boulot, j'en ai rien à foutre de tes vignes" [qui seraient détruites par les fûts d'uranium]. Il y a une période, on les aurait poussés, les fûts d'uranium, mais là, non. Il faut vivre avec son temps. Il y en a assez qui essaient de détériorer l'image des organisations syndicales pour ne pas en rajouter" ${ }^{15}$. En leur ménageant des espaces d'expression spécifiques (militants) et réservés, les responsables syndicaux soumettent ainsi les ressources corporelles du groupe ouvrier à l'apprentissage progressif des règles du jeu, de façon à les mobiliser au service de la cause ou à les ritualiser. En ce sens, on pourrait qualifier les actions spectaculaires, et ce contrairement à une représentation spontanée, d'"institutions servant de soupapes de sécurité", qui "orientent certains sentiments d'hostilité sur des objets de substitution ou qui provoquent des soulagements "cathartiques"'155. Cet exemple a enfin le mérite de montrer combien la violence peut être retravaillée, ritualisée et, en quelque sorte, pacifiée.

\section{NOTES}

1. Michel T., typographe, aujourd'hui retraité, interview du 19 avril 1990, Paris.

2. Voir par exemple les ouvrages de Claude Harmel, en particulier "Les leçons de la manifestation communiste du 23 mars", in Est et Ouest, n 628, mai 1979, p 4-9 ; La CGT à la conquête du pouvoir, Paris, Bibliothèque d'histoire sociale, 1983 ; Francis Bergeron, Le syndicat du livre, ou la mainmise communiste sur la presse, Paris, Ed. Difralivre, 1989.

3. Sur la notion de répertoire d'action, voir Charles Tilly "Les origines du répertoire de l'action collective contemporaine en France et en Grande-Bretagne", in Vingtième siècle, $\mathrm{n}^{\circ} 4$, octobre 1984, $\mathrm{p}$ 89-104.

4. L'Humanité du 23 mai 1990.

5. Sur le thème des classes dangereuses, voir Louis Chevalier, Classes laborieuses et classes dangereuses, Paris, Livre de Poche, 1978 (1ère éd. 1958) et Susanna Barrows, Miroirs déformants, Paris, Aubier, 1990 (Ed. originale 1981). Pour une illustration des "anachronismes" véhiculés par le service d'ordre cégétiste, voir Isabelle Sommier "CGT : du service d'ordre au service d'accueil", à paraitre dans Genèses, juin 1993.

6. Au sens où l'entend Erving Goffman in La mise en scène de la vie quotidienne, Paris, Ed. de Minuit, 1973 (Trad.), Tome 1, p 20-21.

7. Définition classique de T. R. Gurr et H. D. Graham in The History of Violence in America, New York, Bantham Books, 1969, p XXXII.

8. Cette approche de la violence est privilégiée par Yves Michaud, qui considère qu'« il $\mathrm{y}$ a violence quand, dans une situation d'interaction, un ou plusieurs acteurs agissent de manière directe ou indirecte, massée ou distribuée, en portant atteinte à un ou 
plusieurs autres à des degrés variables soit dans leur intégrité physique, soit dans leur intégrité morale, soit dans leurs possessions, soit dans leurs participations symboliques et culturelles ", in Violence et politique, Paris, Gallimard, 1978, p 20. Rappelons également Charles Tilly pour lequel « la violence apparait plutôt comme le sous-produit d'actions et d'interactions qui manifestent une volonté d'agir en fonction d'intérêts contradictoires, mais qui n'ont pas de fondement violent », in op. cit., p 89-104.

9. Respectivement in Longwy, immigrés et prolétaires 1880-1980, Paris, PUF, 1984, p 329 et in Faire l'opinion, Paris, Ed. de Minuit, 1990, p 215.

10. Expression de John H. Goldthorpe. John H. Goldthorpe et al., L'ouvrier de l'abondance, Paris, 1968, Seuil, 1972, p 5.

11. Sur cette question, voir Richard Hoggart, La culture du pauvre, Paris, Ed. de Minuit, 1970 ; Michel Verret, La culture ouvrière, Saint Sébastien, ACL, 1988.

12. Extrait d'une conférence organisée par la revue Economie et Politique, le 15 mars 1955, repris in Claude Poperen, Renault, regards de l'intérieur, Paris, Ed. sociales, 1983, p 183.

13. Voir notamment Richard Hoggart op. cit. ; Paul Willis, "L'école des ouvriers", in Actes de la recherche en sciences sociales, $n^{\circ} 24$, novembre 1978, p 50-61.

14. Expression d'Olivier Schwartz in Le monde privé des ouvriers, Paris, PUF, 1990, $\mathrm{p}$ 298. Pour des exemples, voir $\mathrm{p} 300$ et suivantes.

15. Cette revendication de la virilité par l'obscénité serait, pour Michel Verret, la "dénégation paroxystique d'une impuissance vérifiée partout ailleurs qu'au lit », in La culture ouvrière, Saint Sébastien, ACL, 1988, p 114. Sur cette question, voir également Richard Hoggart, op.cit., chapitre 8. Gérard Mauger émet à ce sujet une hypothèse très stimulante : « Il se pourrait que la défaite dans "le combat" de classe et le reflux des "luttes" sociales d'une part, la dévalorisation de la force physique comme force de travail, d'autre part, poussent au repli des démonstrations de virilité dans les usages de la force physique comme force de combat et dans l'affirmation -proprement sexuellede la virilité », in Les ouvriers : un monde "dé-fait", texte à paraître. Qu'il soit ici vivement remercié de nous avoir permis de lire cet article avant sa parution.

16. Sur ce sujet, cf. Michel Verret, La culture ouvrière, Saint Sébastien, ACL Ed., 1988 ; Florence Weber, Le travail à-côté, étude d'ethnographie ouvrière, Paris, Ed. de l'EHESS, 1989.

17. Sur l'opposition eux/nous, voir Richard Hoggart La culture du pauvre, Paris, Minuit, 1970, p 115 et suivantes (Ed. originale 1957).

18. Exception faite de l'affaire des "dix de Billancourt" qui répondait nettement à des objectifs stratégiques d'ordre politique et provoqua d'ailleurs des dissensions à l'intérieur de la CGT.

19. Crise économique et conflits sociaux, Paris, L'Harmattan, 1979, p 148.

20. Dossier des travailleurs du Parisien Libéré, Le putsch d'Amaury, Paris, Ed. sociales, 1976, p 152.

21. Cf. par exemple le témoignage d'un docker de Dunkerque : « Nous sommes dockers depuis quatre générations. Ca me révolte de savoir qu'on va nous chasser comme des intrus. » in L'Humanité du 13 août 1992. Sur le conflit lorrain, voir notamment Claude Durand, Chômage et violence, Paris, Galilée, 1981 et Christian de Montlibert, op. cit..

22. Robert H., responsable cégétiste, interview du 23 juillet 1992, Cléon.

23. Jean-Pierre B., interview du 23 juillet 1992, Cléon. Noter la métaphore sportive dans cette description.

24. Michel T., interview du 19 avril 1990, Paris. 
25. In La culture ouvrière, Saint-Sébastien, ACL, 1988, p 30.

26. Jean Pierre B., interview du 23 juillet 1992, Cléon.

27. Serge L., interview du 24 mai 1990, Paris.

28. Remise de soi convertie positivement dans la discipline militante, c'est-à-dire la soumission au nom des intérêts supérieurs du groupe. Au-delà du rapport de délégation syndicale, cette remise de soi est facilitée d'un côté, par le caractère "militaire" des opérations spectaculaires qui "justifie" l'obéissance, de l'autre, par l'autorité dont jouit "le chef" sur ses troupes.

29. Maurice L., interview du 19 avril 1990, Paris.

30. Eric Hobsbawm, "Sexe, symboles, vêtements et socialisme", in Actes de la recherche en sciences sociales, $\mathrm{n}^{\circ} 23$, septembre 1978, $\mathrm{p} 18$.

31. Maurice Agulhon, "Propos sur l'allégorie politique. En réponse à Eric Hobsbawm", in Actes de la Recherche en Sciences Sociales, $\mathrm{n}^{\circ} 28$, juin 1979, p 32.

32. Le corps redressé, Paris, Jean-Pierre Delarge, 1978, p 189.

33. Voir Louis Chevalier, Classes laborieuses et classes dangereuses, Paris, Livre de poche, 1978 (1ère éd. 1958), p 678-682 et Gérard Leclerc, L'observation de l'homme, Paris, Seuil, 1979, p 216-258.

34. Jean-Jacques Courtine et Claudine Haroche, Histoire du visage, Marseille, Rivages, 1988, p 275.

35. Voir par exemple Arthur B. Shostak et William Gomberg, Blue-Collar World, Englewoods Cliffs, Prentice Hall, p 57-60.

36. Jean-Pierre B., interview du 23 juillet 1992, Cléon.

37. La "baston" crée ainsi dans les bandes de jeunes de milieux populaires une "ambiance" qui, en même temps qu'elle met à l'épreuve le nouveau venu et marque la cohésion du groupe, s'oppose aux Autres dans un rapport immédiat de corps-à-corps. Sur ce sujet, nous renvoyons le lecteur à François Dubet, La galère, Paris, Fayard, 1987, en particulier $\mathrm{p}$ 184-212 et aux recherches de Gérard Mauger, notamment in Actes de la recherche en sciences sociales, $n^{\circ} 50,1983$ et "La politique des bandes", in Politix, $\mathrm{n}^{\circ} 14$, deuxième trimestre 1991, p 27-43.

38. Jean Baudrillard, Le système des objets, Paris, Denoël-Gonthier 1972 (1ère éd. 1968), p 57. Sur la diffusion de ces codes de conduite, voir Norbert Elias, La civilisation des moeurs, Paris, Hachette, 1969.

39. Réflexions sur la violence, Paris, Seuil, 1990 (1ère éd. 1908), p 66-80 et p 177-196.

40. Philippe Ariès et Georges Duby (Dir.), Histoire de la vie privée, Tome 4, Paris, Seuil, $1987, \mathrm{p} 435$.

41. In La distinction, Paris, Ed. de Minuit, 1979, p 447.

42. In op.cit., p 84.

43. In Le monde privé des ouvriers, Paris, PUF, 1990, p 294. Ne s'agit-il pas plus d'un goût du risque que d'une fascination de la mort?

44. Ibid, p 299.

45. Ibid, p 298.

46. Alain Finkielkraut "La nostalgie de l'épreuve", in Le genre humain, n¹0, 1984, p 59.

47. Jean D., interview du 24 avril 1992, Paris.

48. In op. cit., p 152.

49. In op. cit., p 58.

50. Rapporté in Gérard Noiriel, Vivre et lutter à Longwy, Paris, Maspéro, 1980, p 90. 
51. Sur cette distinction, voir Leonard Berkowitz (eds.), Roots of Aggression. A Reexamination of the Frustration-Aggression Hypothesis, New York, Atherton, 1969 et Philippe Braud, Le jardin des délices démocratiques, Paris, PFNSP, 1991, p 141-156. 52. Maurice L., responsable du service d'ordre cégétiste, interview du 23 mai 1990, Paris.

53. Maurice L., interview du 19 avril 1990, Paris

54. Jean D., interview du 24 mai 1990, Paris

55. Lewis Coser, Les fonctions du conflit social, Paris, PUF, 1982, p 166

\section{RÉSUMÉS}

Depuis une quinzaine d'années, la CGT organise régulièrement des "actions spectaculaires" voire des "actions commandos", qui engagent un petit groupe d'individus fortement encadrés dans une mise en scène guerrière dirigée contre les symboles de l'adversaire étatique ou patronal : occupations de locaux, blocages des voies routières ou ferroviaires, assauts contre des entreprises, etc. Ces opérations interprétées d'un point de vue externe comme une conséquence perverse de la routinisation des manifestations ouvrières ou comme un palliatif à la crise de la syndicalisation, revêtent un intérêt fondamental pour l'étude de la violence dès lors que l'on attache à leur dynamique interne. Elles mobilisent en effet les valeurs constitutives de l'ethos ouvrier, au premier rang desquelles se trouve la virilité - et ses multiples déclinaisons : culte de la force physique, valorisation de la force de combat, camaraderie masculine, solidarité dans la lutte... -, pour les soumettre à des contraintes et à des codes leur permettant de rester socialement acceptables. Ce déploiement de force, théâtralisé et sous contrôle, montre combien la violence peut-être retravaillée, ritualisée et, en quelque sorte, pacifiée.

\section{INDEX}

Index géographique : France

Mots-clés : culture(s), genre, mouvements politiques, Mouvements sociaux, sociologie

Index chronologique : 20ème siècle 